\title{
Group technology in the flexible manufacturing system
}

\author{
Peter Kostal ${ }^{1, *}$, Andrea Mudrikova ${ }^{1}$, and David. Michal ${ }^{1}$ \\ ${ }^{1}$ Slovak University of Technology, Faculty of Materials Science and Technology, J. Bottu 25, 91724 \\ Trnava, Slovak Republic
}

\begin{abstract}
Flexible manufacturing systems enable rapid changes in production. These changes may be due to changes in the product itself, such as different variations of the product or changes in conditions in the production itself. Currently, most products are designed on a computer using a variety of CAD software. Using sophisticated CAD tools, the resulting 3D product model can include not only the geometric product data but the process plan and technology data. Group technology is very often used to quickly create a technological process. Where, based on similarity, parts are assigned to individual groups and each group has the same basis for the technological process.
\end{abstract}

\section{Introduction}

Manufacturing is realized in the environment of high global competition and frequent changes in consumer requirements also put significant pressure on manufacturers to make changes in their production strategies. Manufacturers must be able to anticipate market changes at least to a certain level to respond satisfactorily. Conventional production systems with a centralized structure are not able to react flexibly enough to rapid changes and therefore there are significant changes in the production strategy of manufacturers. This trend is most pronounced in companies seeking to maintain their international competitiveness in an unstable market. Various new forms of organizational structures have been created, such as expanded enterprises, virtual enterprises, virtual organizations, a network of organizations, supply chain management, enterprise grouping, and production networks [1].

Currently is automation very important tools to ensure higher production quality and flexibility. This is the way to improve the manufacturer competitiveness in the globalized market.

In recent years, new definitions of production concepts have been developed. In the 1980s was introduced the concept of Flexible Manufacturing Systems (FMS) to produce a family of products with similar dimensions and shapes. At present, the lack of possibilities to convert such a system to new products family has become a major problem in further improving production processes. At present, the main goal is to change rapidly to start new products or to respond in case of failure in the shortest possible time. This means that in the current production the limiting factor is often the time.

\footnotetext{
*Corresponding author: peter.kostal@stuba.sk
} 
Intelligent Manufacturing Systems (IMS) not only offer flexibility and reconfigurability, but this concept also brings the ideas of software intelligence to manufacturing systems. Such software intelligence manifests itself in various areas such as autonomy, decentralization, flexibility, reliability, efficiency, learning and self-regeneration [2].

Current production is characterized by the trend of production expanding and the innovation cycle shortening. New products have a new shape, material, and function. The production strategy based on a traditional functional production structure changed to production using flexible production cells and lines. At present, the production by automated production systems is one of the most widespread production philosophies [3].

Improvement of the manufacturing responsibility we can achieve by several ways. One of them is shortening the time needed to preparation of the manufacturing. Important part of manufacturing preparation time are consumed by preparing the drawings and process plans. Integrating the $3 \mathrm{D}$ model of product with real-time manufacturing process information is an important prerequisite for improving product quality, reducing costs, and shortening the manufacturing cycle [4].

Drawings are traditionally used for communication in the industry. They are the clearest way to transmit information about what to do. In general, they are considered a universal graphic language. The purpose of the technical drawing is to transmit, check and maintain the product definition in an accurate and clear manner without the risk of misinterpretation or assumption. Technical drawings allow for visual abstraction, display the product in a clear and efficient way, expressing all facts completely and explicitly [5].

In base of drawings and knowledge about a manufacturing technologies, manufacturing devices, tools and fixtures, we can do the process plan for the given part manufacturing. The manufacturing process plan creation is so complex task. This complex operation can be realized easier and faster by use of some ideas from field of group technology. The base idea is in preparation of imagined part (most complex part MCP) which contain the all surface what is possible to manufacture by given combination of machine, tools and fixtures. For this MCP we can prepare the process plan template. In this template will by placed the operations, manufacturing conditions and other variables needed to manufacturing each surfaces.

All real part will contain only subset of the MCP surfaces. And all real process plan will be only subset of the MCP process plan.

In this article will subscribe this process for the turning machine from our flexible manufacturing system (Fig. 1).

\section{Turning machine EMCO TURN 105}

The turning machine EMCO TURN 105 is part of the flexible manufacturing system (FMS), which is located in the laboratory of our institute. The Flexible Manufacturing System with Robotic Operation for a Drawingless Manufacturing Environment (FMS) is represented by the Computer Integrated Manufacturing (CIM) model. It allows a systemic approach to production planning and control. The aim is to gain experience in these areas at the level of the production system as a whole.

The entire FMS (all manufacturing and handling equipment) contains a communication structure based on modern industry standards. This communication structure is also compatible with other industrial devices and enables seamless data transfer. One of the main conditions that were defined as the characteristics of FMS is the ability to cooperate with the CATIA CAD system available at our institute. Also, this system will need to work with other CAD software systems. This cooperation is particularly important in terms of the ultimate goal of the project: "drawingless production" [6].

The turning machine is operated by industrial robot. As a fixture is used the hydro pneumatic vice with clamping diameter $28-31 \mathrm{~mm}$. This lathe are equipped by 8 position 
revolver head for tool changing. Maximal turning length is $240 \mathrm{~mm}$, but the real is smaller, only $(121 \mathrm{~mm}$ ) because the revolver head must be able change the axial tools (drills) too. Maximal turning diameter is $140 \mathrm{~mm}$.

Must take a mind for this physical limitations of machine in time of preparing the process plan for real manufactured part. Other limitation are the tools what are equipped in the revolver head.

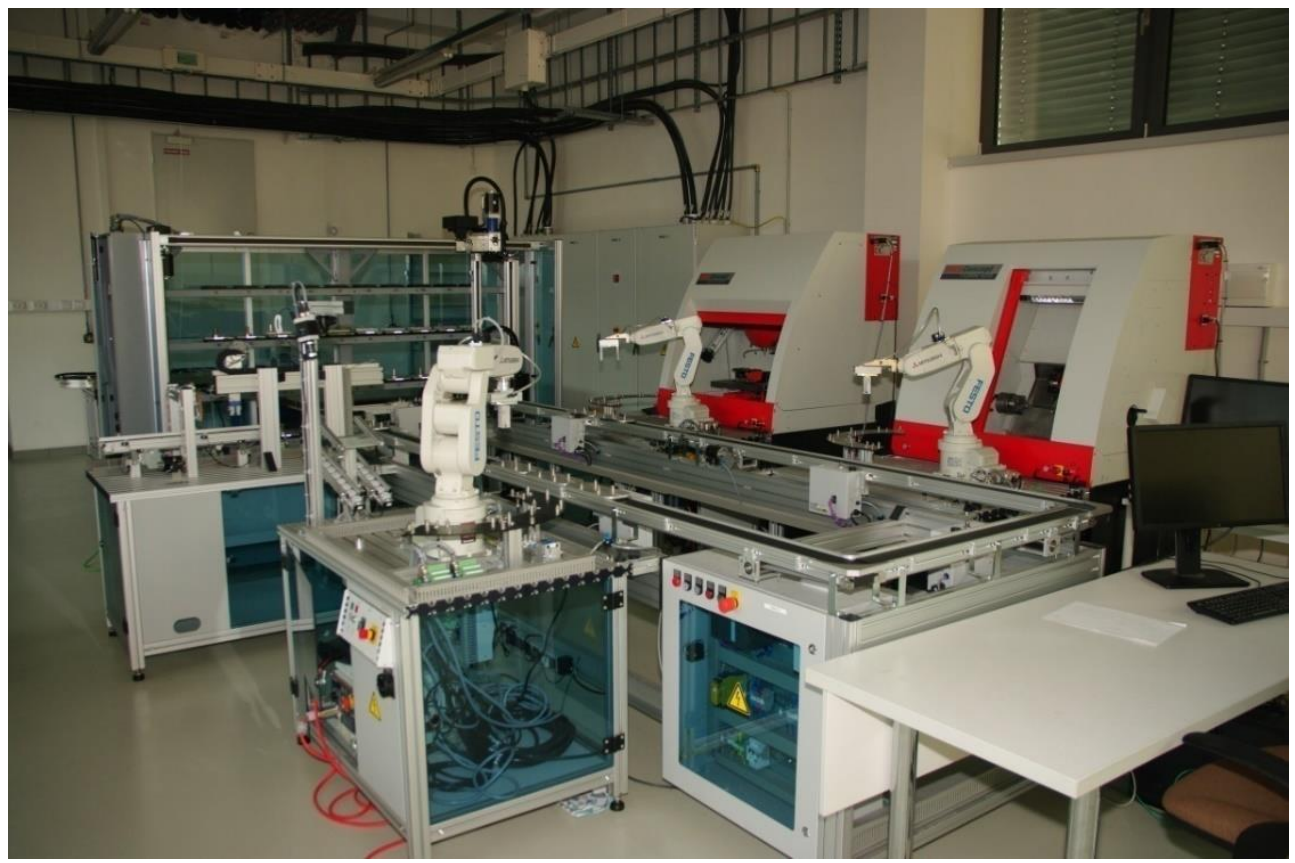

Fig. 1. Flexible manufacturing system

The revolver head are equipped by tools only in position 1-5. In position 1, 2 are two different kind of turning tools, in position 3 are equipped by centering drill, position 4 is the helical drill, and position 5 are occupied by countersink drill (Fig. 2).

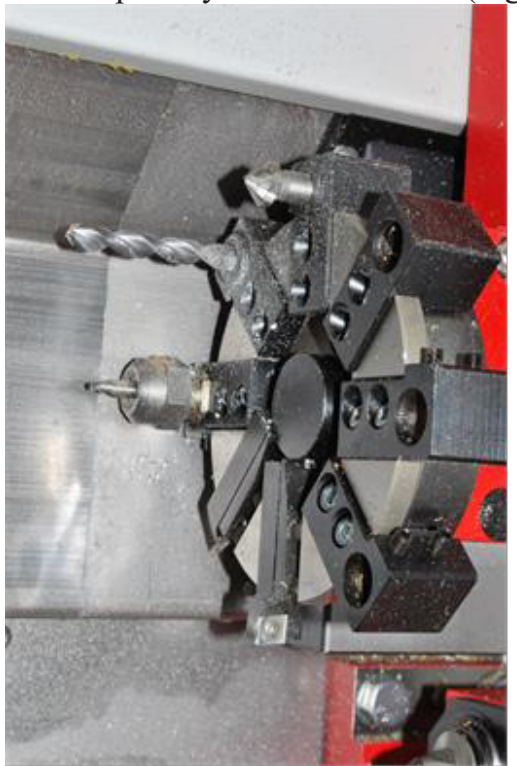

Fig. 2. Revolver toolholder head at turning machine 


\section{Possibilities to surface creation by cutting}

All parts consists of the final number of defined surfaces. In order to produce a specific part, we must take into account the appropriate combination of machine tool - motion and cutting tool. In some cases of surfaces, it is necessary to adjust another surface (such as thread cutting). Therefore, it is essential to pay increased attention to production order of surface in the production process.

The surfaces creation at the part by turning processes are possible three ways [7]:

- $\quad$ Point + movement - usually longitudinal turning the rotational surfaces, transverse turning the heading surfaces;

- $\quad$ edge + movement - usually grove turning;

- $\quad$ surface + movement - drilling operations in general.

In the Table 1 can see the general cases of surface creation by turning operation by given combination of machine and tools.

The first tool able to create the surfaces only as a point (tool tip) + movement. This tool are used for longitudinal turning, or for face turning.

The second tool can create the surface usually as a point + movement. This case is usable for longitudinal turning, but this tool can use a grove turning too. This case is the edge+move and final surface are footprint of edge shape, in this case is the $\mathrm{V}$ grove. This means, that the tool geometrical characteristics are partially copied into geometrical characteristics of machined part.

The third, fourth and fiveth tool are drilling tools, so they create the new surface by method surface+movement. This means that the geometrical characteristics of tool are almost fully copied into geometrical characteristics of created surface.

Table 1. Surface creation by several tools at turning machine.

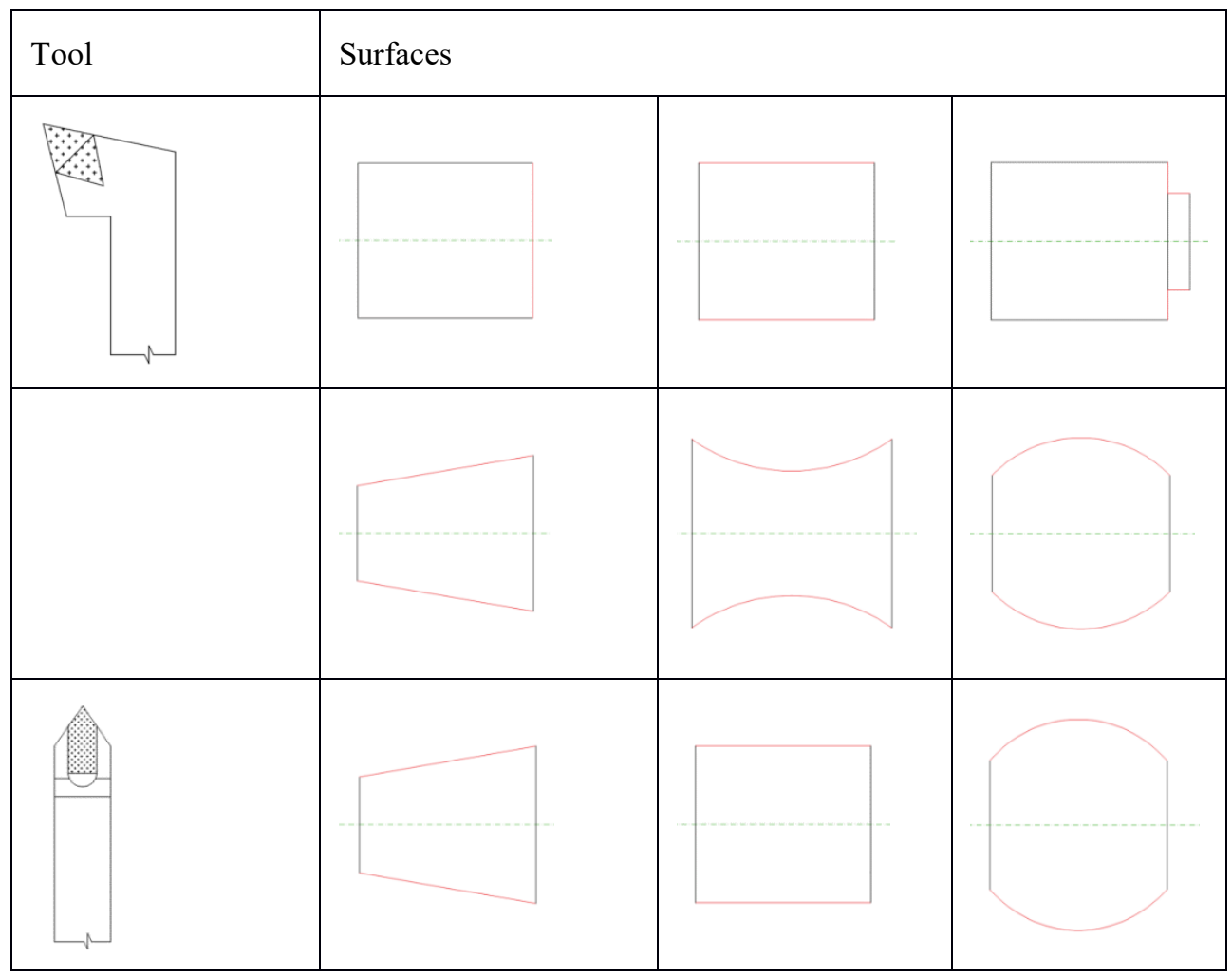




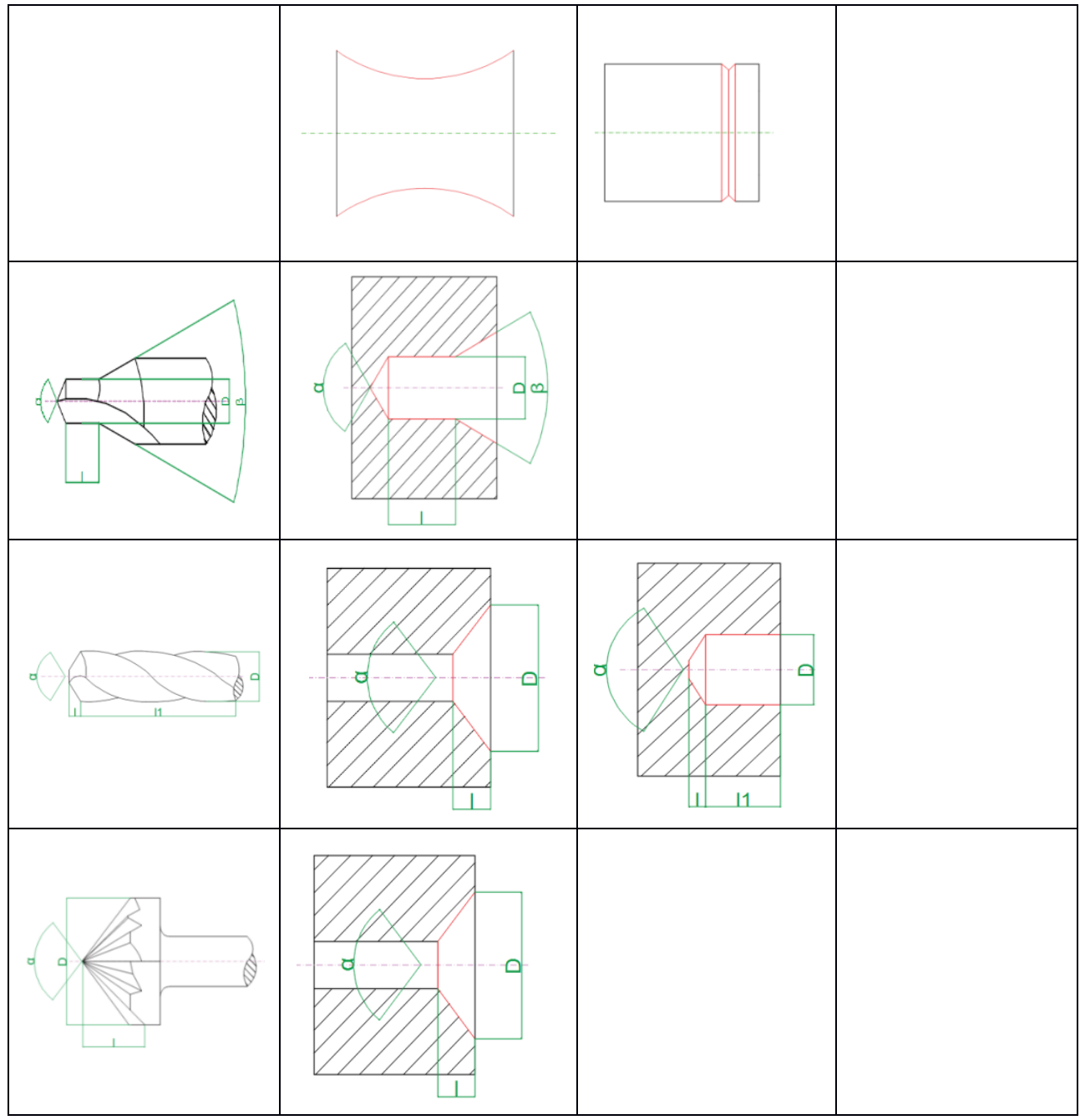

By combination of all manufacturable surfaces from table we create the MCP (Fig. 3). This part contain all of the possible surfaces what we can realize by given machine and given tools. This part is only imaginal, all real part will contain only a subset of surfaces from this imaginal part.

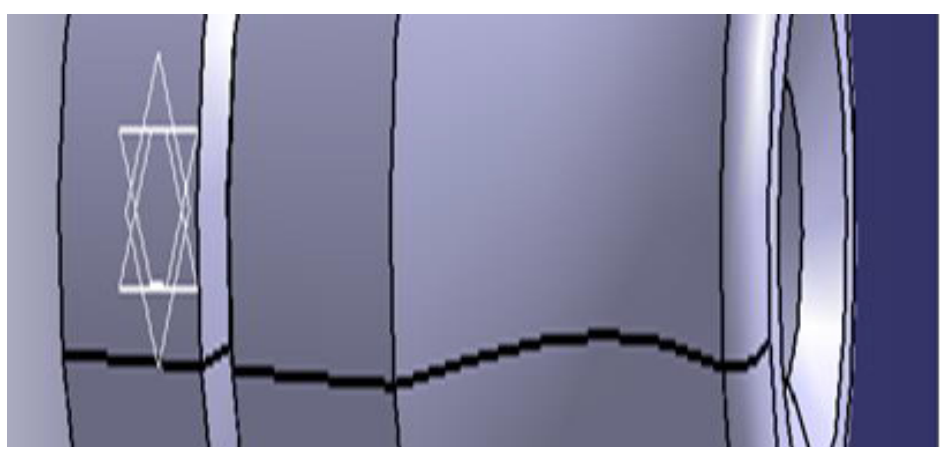

Fig. 3. MCP model created by combination of all manufacturable surfaces from table 1. 


\section{Group technology and MCP process plan}

In the base, there are two kinds of CAPP systems: variant and generative. The variant approach is similar to the manual creation of technological processes, that is the way the process planer uses it. In this case, the process plan for the new part is obtained by making minor changes to the process plan that is already available for a similar part. The basis is a database of existing technological procedures and process plans for existing parts and the similarity of the new part with an existing one.

A generative approach generates a new process plan for each part based on the constraints inserted and the new part model. Generation then proceeds usually without the process planner interfering with the generation process. In this case, sophisticated mathematical algorithms are used based on various mathematical tools and procedures such as decision tables, decision trees, expert systems, genetic algorithms, etc.

There are also semi-generational (or hybrid) CAPP systems in which some tasks performed by technology in a variant system are automated, and at the same time a part of the process is generated (usually the part that cannot be created in the variant system for some reason) parts can then be modified.

To create the right process plan, it is necessary to provide a detailed analysis of the part, its shape, dimensions, as well as the dimensional and geometric tolerances or surface roughness and the like. For this reason, the data entering the CAPP must be in the form of a suitable mathematical model. When using modern control systems of production machines, which enable them to work with so-called production features (recess, thread, drilled hole,...), it is advantageous to use these production features directly in the compilation of the technological process. In such a case, it is even very common that the entire manufactured part can only be manufactured using predefined manufacturing features in which only the current parameters are entered. In the case of material removal manufacturing processes, these features correspond to the machining properties that are parts of the work piece affected by the machining operation [1].

To achieve efficient production, it is necessary to realize the balancing of production despite high product diversity. This means that the various products are grouped into a manageable number of product groups according to their manufacturing similarity. A large number of different products are commonly found in practical cases. These must be grouped by a large number of attributes. These attributes can be geometric (shape, size), technological (production method), material (steel, aluminum,...), and of course various others. Such attributes are a specification of the selected grouping criteria. Usually, there is no pre-determined number of part groups or pre-classified objects [8].

Group Technology (GT) is a methodology that uses the principle of a variant design of the technological process. It uses mainly geometric and technological similarity between machine parts or functions. When creating individual groups of parts, it is necessary to take into account the relevant properties of the part. The basic requirement of the GT method is that the information is known in advance and remains constant throughout its use. On the other hand, current production requires flexibility with less regard for the functions used for grouping [9].

As already mentioned, the component groups are formed by identifying similarities. These similarities can vary and are reflected in the different grouping criteria. Attributes focused on geometry and production technology generally represent the two most common categories of grouping criteria applicable to the formation of a product group. While the geometry criteria describe the products concerning their geometric and physical characteristics, the production criteria describe the similarities regarding the production sequence and other manufacturing requirements. These technological similarities need to be known for the production of products of one group in order without or with minimal time loss (eg caused by changes). 
Adequate grouping criteria to form product groups to balance production are the sequence of operations, required equipment and personnel, process times, set up times for replacement and sharing of identical components, parts or raw materials. [8]

By using the methods of GT we can prepare the process plan template for manufacturing the all real part on base of the MCP. This template will contain the manufacturing data for all surfaces in the MCP. These data in the template contain specification of the surface, specification of the tool and turning operation.

In case of manufacturing the real part (subset of $\mathrm{MCP}$ ) we select from this template the appropriate subset of surfaces (subset telling about the surfaces, what are content in our real part) as a raw process plan, and fill the real part specific data.

Current programs for generating technological processes are mainly based on the principles of group technology. These programs have in their library stored templates of technological procedures for individual product groups.

During the generation of the technological process, the specified values in the template (eg length, diameter, ...) are filled with actual values from the part drawing or directly from $3 \mathrm{D}$ model of the part. This means that the technological procedure for different parts of a given group is always the same, only the individual parameters of the part in this procedure change. Alternatively, some operations that did not have filled parameters are omitted.

Once it is necessary to produce a product that cannot be assigned to any existing group, it is necessary to create a new group and at the same time define a new template for the technological process.

The raw process plan contain the sequence of operations for creating the real part by a given machine and given tools. But in this raw process plan are not determined the real part specific data. These data such as the cutting conditions or part dimensions will be filled in the next three steps.

In first step we fill the part specific geometrical data such as a dimensions and positions of the each surface (type of surfaces are given in the raw process plan).

In second step we give the technological specific data. They are data about part material, cutting material and other technology specific data. These technological data must be chosen carefully. More about a correct choosing of technological data is at the literature [10].

In third step are calculated the specific cutting parameters on base of filled data. All of the filled and calculated parameters are filled into the part specific process plan.

At our institute are working on an algorithm that will use the principle of group technology but will not work with groups of parts. It will work with individual production areas. This means that instead of the template of the technological process of production of the whole component (group of components), it will work with templates for the production of individual areas on the given component. In each such template there will be different possibilities of manufacturing the given surface on base of manufacturability. According to the entered criteria is then chosen the appropriate method of production of the area.

\section{Conclusions}

Nowadays, due to the shortened product life cycle, great competition, and diverse customer requirements, companies are forced to gradually change their production philosophy from mass production to small series with a wide range of products. There are many problems associated with this change, especially in the areas of inventory planning, organization of production, rationalization of work. These related problems are described, for example, in [10, 11].

This paper provides a brief overview of how to use the foundations of surface creation theory by machining and the idea of group technology in modern flexible manufacturing systems. 
The paper provides the basis for a wider research project that will contribute to enabling the transition to smarter production systems and smarter production in these systems. Another possible course of research is to improve what has been described in this article and to use the simulation to examine different production scenarios.

This paper was written thanks to the national KEGA Grant: 021STU-4/2018 - Development of a laboratory for the design and maintenance of production systems supported by the use of Virtual Reality

\section{References}

1. A. J. Álvares, J. C. E. Ferreira, and R. M. Lorenzo, J. Intell. Manuf., 19, no. 6, p. 643 (2008)

2. F. a Almeida, P. a Dias, G. a Gonçalves, M. b Peschl, and M. c Hoffmeister, Int. J. Ind. Eng. Comput., 2, no. 4, pp. 873-890 (2011)

3. P. Kostal and A. Mudrikova, "Laboratory of Flexible Manufacturing System," AMR, no. 429, pp. 31-36 (2012)

4. D. N. Šormaz, J. Arumugam, R. S. Harihara, C. Patel, and N. Neerukonda, Robot. Comput.-Integr. Manuf., 26, no. 6, pp. 583 - 595 (2010)

5. V. Quintana, L. Rivest, R. Pellerin, F. Venne, and F. Kheddouci, Comput. Ind., 61, no. 5, pp. 497-508 (2010)

6. P. Koštál, D. R. Delgado Sobrino, R. Holubek, and R. Ružarovský, Appl. Mech. Mater., 693, pp. 3-8, (2014)

7. J. Békés, Analýza a syntéza strojárskych objektov a procesov. ALFA-vydavatel'stvo technickej a ekonomickej, (1986)

8. F. Bohnen, T. Maschek, and J. Deuse, "Leveling of low volume and high mix production based on a Group Technology approach,” CIRP J. Manuf. Sci. Technol., 4, no. 3, pp. $247-251$ (2011)

9. D. Ben-Arieh and R. Sreenivasan, "Information analysis in a distributed dynamic group technology method,” Int. J. Prod. Econ., 60-61, pp. 427-432 (1999)

10. J. Kundrák, I. Sztankovics, and K. Gyáni, "Analysis of the Theoretical Values of Several Characteristic Parameters of Surface Topography in Rotational Turning"

11. P. Tamás, "Application of Value Stream Mapping at Flexible Manufacturing Systems," Key Eng. Mater., 686, pp. 168-173 (2016)

12. G. Kovács and S. Kot, "Facility layout redesign for efficiency improvement and cost reduction,” J. Appl. Math. Comput. Mech., 16, no. 1, pp. 63-74 (2017) 